\title{
Comparative Study on Fire Protection Standards of Tunnel Engineering at Home and Abroad
}

Guohui Li, Weiping Han, Lizeng Zhao

Tianjin Fire Research Institute of Ministry of Public Security, Tianjin 300381, China

\section{国内外隧道工程防火技术标准对比研究}

李国辉, 韩伟平, 赵力增

公安部天津消防研究所, 天津 300381 , 中国

\begin{abstract}
Fire is one of the greatest threat to the tunnel. A large number of research results of metro and tunnel fire prevention technology have been accumulated in Europe, America and Japan. In these country and region, a series of standard have been applied to tunnel engineering. Based on the standards of UIC, CEN, NFPA, Europe and Japan, et al, the paper focuses on the status and dynamics of fire protection standards of highway, railway and subway tunnels. Some suggestions have been proposed by comparing the fire protection standards at home and domestic.
\end{abstract}

Keywords:tunnel; fire protection; standards

\section{摘要}

火灾事故是隧道最大威胁之一。在地 铁及隧道防火方面, 欧美日等研究成果丰 硕, 并形成一系列标准规范。以国际铁路 联盟、欧洲标准化委员会、美国消防协会 等标准化组织以及欧洲和日本等主要国家 和地区发布的标准规范为研究对象, 研究 公路、铁路和地铁隧道防火技术标准现状 与动态, 综合分析我国标准制修订现状, 通过对比提出我国标准规范制修订建议。 关键词：隧道；防火；标准规范
1. 国内地铁及隧道工程防火技术标准现 状

我国现行地铁及隧道防火技术标准规 范包括《地铁设计规范》(GB 50157-2013) 《地铁安全疏散规范》(GB/T 33668-2017)、 《铁路隧道设计规范》( TB 10003-2016)、 《公路隧道设计规范》( JTG D70-2-2014)、 《建筑设计防火规范》(GB 50016-2014)、 《铁路工程设计防火规范》( TB100632016) 等 30 多项 ${ }^{[1-6]}$ 。《建筑设计防火规范》从消 防给水、灭火设施、通风排烟、火灾自动 报警系统、供电等等领域提出了城市交通 隧道通用防火设计要求, 不涉及地下铁路 隧道、管线和电缆隧道。《地铁设计规范》 主要针对地铁站, 缺少对区间隧道的防火 规定。《铁路隧道设计规范》将铁路隧道分 为四类，但没有明确隧道防火设计要求。

新发布的《铁路工程设计防火规范》修订 了铁路隧道紧急救援站消火栓和细水雾灭 火系统设计要求, 规定了消防给水设施和 灭火系统、照明和疏散指示标志等设计要 求, 但规定较笼统, 仍不能满足实际需求。 《公路隧道消防技术规范》针对交通隧道 的设计、施工和验收, 规定了火灾设计规 模、消防设施配置要求、I 级隧道泡沫喷 淋和细水雾系统设置要求, 但作为地方标 准尚不具备强制性和普适性 ${ }^{[7]}$ 。

总体来看, 我国隧道防火技术标准发 展滞后, 技术条款尚待细化, 在地铁及隧 
道防火设计方面函需出台专项国家标准。

\section{2. 国内外地铁及隧道防火技术标准对比}

\section{1 公路隧道防火关键技术指标对比}

\subsection{1 公路隧道分级}

我国行业标准依据长度将非城市公路 隧道分为短隧道 $(\mathrm{L} \leqslant 500 \mathrm{~m})$ 、中隧道 $(500 \mathrm{~m}<\mathrm{L} \leqslant 1000) 、$ 长隧道 $(1000 \mathrm{~m}<\mathrm{L} \leqslant$ $3000)$ 和特长隧道 $(3000 \mathrm{~m}<\mathrm{L})$; 《建筑设 计防火规范》根据隧道用途和长度分为四 类。美国根据长度分为五类: $X$ 类 $\mathrm{L}<90 \mathrm{~m}$ 、 $A$ 类 $90 \mathrm{~m} \leqslant L 、 B$ 类 $240 \mathrm{~m} \leqslant L$ 且任一点至安全 区域距离超过 $120 \mathrm{~m} 、 C$ 类 $300 \mathrm{~m} \leqslant \mathrm{~L} 、 \mathrm{D}$ 类 $1000 \mathrm{~m} \leqslant \mathrm{~L}$ 。日本依据日均车流量及长度按 危险性依次降低顺序分为五类: AA 级、 $\mathrm{A}$ 级、 $B$ 级、 $C$ 级和 $D$ 级。德国根据长度分为 四类, 瑞典根据交通量分为四类, 法国依 据隧道是否位于城市以及长度分为三类, 英国与日本类似分为五类。与国外相比, 我国对隧道的分级依据隧道长度有一定合 理性, 但对非城市公路隧道分类没有综合 考虑交通情况, 安全等级划分相对简单。

\section{1.2 消防设施设置依据}

国外主要依据隧道长度确定是否设置 消防设施, 部分国家考虑因素更为具体, 如日本、挪威、瑞典和英国以交通流量为 依据, 奥地利以隧道潜在危险性为依据, 韩国通过风险分析确定, 法国和德国依据 隧道位置、交通类型、拥堵风险和管理因 素等综合确定。各国都规定了设置消防设 施的最小隧道长度, 具体为: 德国和韩国 要求所有隧道均设置消防设施、美国为 $90 \mathrm{~m}$ 、 日本和瑞典为 $100 \mathrm{~m}$ 、英国为 $150 \mathrm{~m}$ 、法国为 $300 \mathrm{~m}$ 、挪威和欧盟为 $500 \mathrm{~m}$ 。我国对于设置 消防设施的要求根据隧道类别确定, 没有 规定需设置消防设施的最小隧道长度。

\section{1.3 灭火设施}

灭火器: 我国规定隧道内应设置 $A B C$ 类灭火器, 间距不大于 $100 \mathrm{~m}$ 。美国要求在 B、C、D 类隧道内必须设置便携式灭火器, 间距不大于 $90 \mathrm{~m}$, 奥地利规定间距不大于 $250 \mathrm{~m}$ 、法国规定间距不大于 $200 \mathrm{~m}$ 、瑞士要 求间距不大于 150 。
消火栓: 我国规定隧道内设置消火栓, 其中, 单洞双车道隧道的消火栓间距不应 大于 $50 \mathrm{~m}$, 单洞三车道和四车道隧道的消火 栓间距不应大于 $40 \mathrm{~m}$ 。美国规定消火栓间距 不超过 $305 \mathrm{~m}^{[8]}$, 法国规定消火栓间距不大 于 $200 \mathrm{~m}$, 奥地利间距要求为 $250 \mathrm{~m}^{[9]}$, 日本 和韩国均规定消火栓间距不大于 $50 \mathrm{~m}$ 。

固定灭火设施: 《建筑设计防火规范》 未涉及固定灭火系统; 《公路隧道设计规范 第二册 交通工程与附属设施》提出了设置 固定式水成膜泡沫灭火装置设计规定。澳 大利亚要求隧道内优先设置雨淋系统; 日 本最早在公路隧道内设置固定灭火设施, 要求 $\mathrm{AA}$ 类和部分 $\mathrm{A}$ 类隧道设置水喷雾系统; 欧洲国家规定在隧道内设置细水雾系统; 美国 NFPA 502 规定 C、D 类隧道根据情况 设置固定式水基灭火系统, 包括雨淋系统、 水喷雾系统和泡沫灭火系统。

与国外比, 我国对灭火器和消火栓的 规定是合理的, 与国外的差异在于灭火器 设置数量和间距, 以及消火栓的流量、压 力及设置间距等要求。不同国家对于设置 固定灭火系统的规定存在差异, 对于固定 灭火系统的有效性尚缺乏数据支撑。

\subsection{4 疏散与救援设施}

我国《公路隧道设计规范》规定上、 下行分离式独立双洞公路隧道间应每隔 $250 \mathrm{~m}-500 \mathrm{~m}$ 设横向联络通道, 通道宽度不小 于 $2 \mathrm{~m}$, 两端应设防火门。美国 NFPA 502 规 定紧急出口间距不应大于 $300 \mathrm{~m}$, 出口采用 1. $5 \mathrm{~h}$ 的防火门, 疏散通道净宽度不小于 $1.12 \mathrm{~m}$, 采用耐火极限不小于 $2 \mathrm{~h}$ 结构进行 分隔, 装修应达到 A 级。欧盟 Directive 2004/54/EC 规定公路隧道内应设急救站, 并包括至少一部应急电话和两具灭火器, 新建隧道急救站间距不应大于 $150 \mathrm{~m}$, 在用 隧道急救站间距不应大于 $250 \mathrm{~m}^{[10]}$; 新建隧 道单洞车流量超过 2000 辆/日, 必须设置 紧急出口, 在用隧道长度大于 $1000 \mathrm{~m}$, 车流 量大于 2000 辆/日时经评估决定是否需增 设紧急出口, 出口间距不应大于 $500 \mathrm{~m}$, 指 令还规定每 $1500 \mathrm{~m}$ 设置应急横向通道, 每 $1000 \mathrm{~m}$ 设置紧急避难间。与国外相比, 我国 只规定了间距、通道宽度和防火门要求, 
对结构和装修耐火性能无详细规定, 对紧 急服务通道及急救站的规定不够严谨具体。

\section{1.5 火灾探测报警设施}

《公路隧道交通工程设计规范》 ( JTG/T D71 2004) 规定, 隧道、中央控 制室和变配电所设备间应设置火灾探测器, 手动报警按钮设置间距应不大于 $50 \mathrm{~m}$, 火灾 探测器的影响时间不应大于 $60 \mathrm{~s}$ 。

NFPA 502 规定 B、C、D 类隧道应设置 火灾探测系统, 并能手动启动, 公路隧道 安装水基灭火系统时, 应装自动火灾探测 系统。B、C、D 类隧道辅助空间及附属区域 应设火灾探测系统。其中, 手动火灾报警 按钮每隔 $90 \mathrm{~m}$ 设置一个, 自动火灾探测系 统应能识别 $15 \mathrm{~m}$ 内的火灾。火灾探测系统 应该能够在 $5 \mathrm{MW}$ 或少 $90 \mathrm{~s}$ 内或 $3 \mathrm{~m} / \mathrm{s}(590$ fpm) 空气速度中检测隧道火灾事故。

欧盟规定所有隧道应设置火灾探测系 统, 但可不设控制中心, 其他无详细规定。

德国规定隧道长度大于 $400 \mathrm{~m}$ 或设置机 械通风时应设置火灾探测系统, 探测系统 应能在 $6 \mathrm{~m} / \mathrm{s}$ 的气流速度下在 $30 \mathrm{~s}$ 内探测出 $5 \mathrm{MW}$ 的火灾, 探测保护距离不大于 $50 \mathrm{~m}^{[11]}$ 。

与国外相比, 我国对探测器要求低, 而对报警系统设计标准规定较为详细。我 国的公路隧道火灾探测报警技术研究起步 晚, 但发展快, 目前正在制定公路隧道火 灾报警专项技术标准《公路隧道火灾自动 报警系统设备技术条件》和《公路隧道消 防技术规范》等, 均详细规定了火灾探测 和报警系统的设计要求, 但尚未发布。

\subsection{6 通风排烟系统}

设置要求: 我国规定大于 $1000 \mathrm{~m}$ 一级 公路隧道, 大于 $2000 \mathrm{~m}$ 二、三、四级公路 隧道, 通行机动车的一、二、三类城市交 通隧道应设机械排烟设施。欧盟规定大于 $1000 \mathrm{~m}$ 且车流量大于 2000 辆/日的隧道设机 械通风系统, 双向隧道或拥堵的单向隧道 通过风险分析决定是否设置纵向通风。美 国 NFPA 502 规定大于 $1000 \mathrm{~m}$ 的隧道必须设 置应急通风系统。与国外相比, 我国的要 求与美国和欧盟基本一致。但我国对于自 然排烟、纵向排烟和横向排烟的规定不够
具体。对于自然排烟, 法国规定大于 $300 \mathrm{~m}$ 的城市隧道, 大于 $500 \mathrm{~m}$ 的非城市隧道, 以 及大于 $1000 \mathrm{~m}$ 的轻交隧道应设置; 德国认 为短隧道设置排烟系统不合理, 规定小于 $400 \mathrm{~m}$ 的隧道不设置排烟系统; 挪威规定小 于 $250 \mathrm{~m}$ 的隧道不设置排烟系统; 英国规定 小于 $300 \mathrm{~m}$ 的隧道仅靠车辆活塞风效应即可 满足气流交换, 可不设置排烟系统。对于 纵向排烟, 法国规定大于 $5000 \mathrm{~m}$ 的单向非 城市隧道、大于 $500 \mathrm{~m}$ 的单向城市隧道、大 于 $1000 \mathrm{~m}$ 的双向非城市隧道应设置, 但双 向城市隧道不设置纵向排烟; 德国规定大 于 $600 \mathrm{~m}$ 的隧道应经风险评估决定是否设置。

临界风速: 我国规定采用纵向排烟的 临界风速在 20MW 火灾时介于 2.0-3.0m/s, 在 30MW 时介于 3.0-4. $0 \mathrm{~m} / \mathrm{s}$, 采用排烟道集 中排烟时, 纵向设计风速不宜大于 $2.0 \mathrm{~m} / \mathrm{s}$; 长度大于 $3000 \mathrm{~m}$ 的城市隧道宜采用纵向分 段排烟或重点排烟, 长度不大于 $3000 \mathrm{~m}$ 的 单洞单向城市隧道宜采用纵向排烟, 单洞 双向交通隧道宜采用重点排烟。Directive 2004/54/EC 规定当设置机械通风系统且不 允许纵向通风时应设置横向或半横向通风 系统; 当双向隧道车流量大于 2000 辆/日， 长度大于 $3000 \mathrm{~m}$ 且有控制中心和横向或半 横向通风时, 应设置排烟阀。NFPA 502 分 别规定了双向车道和单项车道隧道的排烟 模式应满足设计目标, 给出了设计的指导 原则。与国外相比, 美国根据设计目标确 定排烟方式, 依据实际情况计算确定临界 风速, 强调目标导向, 结果更加科学合理。

\subsection{7 疏散指示标志}

疏散指示标志: NFPA 502 规定, 在紧 急出口侧墙上应设置安全疏散指示标志, 间距不应大于 $25 \mathrm{~m}$ 。欧盟 Directive 2004/54/EC 规定在隧道侧墙上设置疏散指 示标志, 间距不大于 $25 \mathrm{~m}$, 高度位于地面以 上 1.0-1.5m 处, 并明确标注到安全出口的 距离。我国《公路隧道设计规范第二册 交通工程与附属设施》规定长度大于 $500 \mathrm{~m}$ 的公路隧道内设置疏散指示标志, 间距不 大于 $50 \mathrm{~m}$ 。对比可见欧美规范对疏散指示标 志间距的规定更为严格。 


\section{2 铁路隧道防火关键技术指标对比}

\subsection{1 铁路隧道防火设计总体要求}

不同国家标准规范针对目标不同。瑞 士制定了隧道总体设计指南, 适用于大于 $25 \mathrm{~km}$ 的新建隧道; 意大利标准规范适用于 5-20km 建成隧道, 以及 $2-20 \mathrm{~km}$ 新建隧道, 对于大于 $20 \mathrm{~km}$ 的隧道应单独研究确定; 德 国标准规范适用于 $1-15 \mathrm{~km}$ 新建隧道, 对于 大于 $15 \mathrm{~km}$ 隧道应单独研究确定; 法国标准 规范适用于 $400 \mathrm{~m}-10000 \mathrm{~m}$ 的新建城市隧道、 客运及混合线路隧道, 大于 $10 \mathrm{~km}$ 的隧道以 及大于 $5 \mathrm{~km}$ 的繁忙铁路线路应单独研究确 定; 英国标准规范适用于浅埋铁路隧道、 大于 $1.5 \mathrm{~km}$ 的地铁隧道和重型铁路隧道、 新建和改建隧道; 国际铁路联盟相关规范 主要针对新建和建成大于 $1 \mathrm{~km}$ 铁路隧道; 我国铁路隧道防火技术相关标准主要针对 $1.0 \mathrm{~km}$ 以上的新建、高速铁路隧道。

总体来看, 铁路隧道相比公路隧道火 灾危险性较小, 对应的防火技术要求也相 对宽松, $1 \mathrm{~km}$ 以下铁路隧道的防火技术要求 不做强制要求。

\section{2.2 灭火设施}

澳大利亚规定隧道内应设便携式手提 灭火器、无压湿式系统消火栓 (间距不大 于 $120 \mathrm{~m}$ ), 无固定灭火设施设置要求 ${ }^{[12]}$; 设 备洞室必须设置自动灭火装置。欧洲铁路 联盟规定隧道内应设置消火栓系统, 间距 不小于 $250 \mathrm{~m}$, 设备洞室内设置自动灭火系 统或手动灭火系统。瑞士规定在隧道口设 置灭火器, 根据实际情况在紧急停车点设 置灭火器。美国规定隧道内应设置便携式 灭火器, 其数量、尺寸、型号及位置应符 合相关规定, 无固定灭火设施设置要求 ${ }^{[13]}$ 。

我国规定长度 $5.0 \mathrm{~km}$ 及以上的客货共 线铁路隧道两端洞口处宜设置高位水池, 并应各设置两部消火栓, 消火栓距洞口距 离不宜小于 $50 \mathrm{~m} 。 5.0 \mathrm{~km}$ 及以上隧道内通风、 电力、电力牵引、通信、信号设备洞室应 设置自动灭火装置, 并应配置 3 具 $4.0 \mathrm{~kg}$ 的 $\mathrm{ABC}$ 干粉灭火器。对比可见, 对于铁路 隧道, 国内外要求基本一致, 对于是否设 置固定灭火系统尚未形成共识, 但对设备
洞室设置灭火设施的要求严于隧道本身。

\subsection{3 疏散与救援设施}

美国规定大于 $610 \mathrm{~m}$ 隧道应配备应急疏 散车, 应急出口最大间距为 $762 \mathrm{~m}$, 门为平 开门或滑动门且最小净宽不小于 $1.12 \mathrm{~m}$; 联 络通道最大间距为 $244 \mathrm{~m}$, 净宽不应小于 $1.12 \mathrm{~m}$, 净高不应小于 $2.1 \mathrm{~m}$ 。澳大利亚规定 应急出口最大间距为 $240 \mathrm{~m}$, 疏散通道最小 宽度为 $0.85 \mathrm{~m}$, 净高不小于 $2.1 \mathrm{~m}$ 。

欧盟规定应急出口最大间距为 $1000 \mathrm{~m}$, 净宽和净高分别不小于 $1.5 \mathrm{~m}$ 和 $2.25 \mathrm{~m}$ 。联 络通道间距不应大于 $500 \mathrm{~m}$, 净宽和净高分 别不小于 $1.5 \mathrm{~m}$ 和 $2.25 \mathrm{~m}$; 大于 $500 \mathrm{~m}$ 的隧道 应设置逃生通道, 宽度不小于 $0.75 \mathrm{~m}$, 高度 不小于 $2.25 \mathrm{~m}$; 应设置救援通道, 宽度不应 小于 $2.25 \mathrm{~m}$, 高度不应小于 $2.25 \mathrm{~m}$ 。

国际铁路联盟规定应急出口间距不应 大于 $1000 \mathrm{~m}$, 疏散通道宽度应大于 $0.7 \mathrm{~m}$, 最佳宽度为 $1.2 \mathrm{~m}$; 络通道间距不大于 $500 \mathrm{~m}$ 。

我国规定时速 200-350km 铁路隧道内 应设置救援通道, 单线隧道单侧设置, 多 线隧道双侧设置, 通道宽度不宜小于 $1.5 \mathrm{~m}$, 净高不应小于 $2.2 \mathrm{~m}$; 最高时速 $160 \mathrm{~km} / \mathrm{h}$ 铁 路隧道救援通道宽度不应小于 $1.25 \mathrm{~m}$ 。联络 通道间距不应大于 $500 \mathrm{~m}$, 净宽不应小于 $2.3 \mathrm{~m}$, 净高不应小于 $2.5 \mathrm{~m}$ 。应急出口净宽 不应小于 $2.3 \mathrm{~m}$, 净高不应小于 $2.5 \mathrm{~m} 。 20 \mathrm{~km}$ 及以上隧道或隧道群应设置紧急救援站, 救援站间距不应大于 $20 \mathrm{~km}$ 。与国外相比, 我国对铁路隧道应急出口的设置间距没有 明确; 联络通道间距设置要求与欧洲基本 一致, 但低于美国标准; 我国尚未规定隧 道内设置应急疏散车, 低于美国标准。

\section{2.4 火灾探测报警设施}

我国规定 $5.0 \mathrm{~km}$ 及以上隧道大型避车 洞内应设事故报警电话。欧盟规定隧道内 外用于人员自救、安全疏散、应急通信、 灭火和救援、以及提供牵引力的技术和设 备洞室均应设置火灾探测器。美国规定随 道变电站和通信室应设置探测系统并与控 制中心相连。澳大利亚规定设备洞室必须 设置火灾探测系统, 大于 $250 \mathrm{~m}$ 隧道应设置 洞口监控系统。与国外相比, 我国缺少详 
细的探测报警系统设置要求。

\subsection{5 通风排烟系统}

澳大利亚规定满足疏散要求且小于 $160 \mathrm{~m}$ 的隧道可不设机械通风, 其他设机械 通风时应满足: 气流可阻止任何位置烟气 回流; 出口通道温度不超过规定; 隧道和 疏散区域气流速度应小于 $12 \mathrm{~m} / \mathrm{s}$; 气流方向 与疏散方向相反; 通风设备具备自动和手 动启动功能; 系统工作时间不应小于 $1 \mathrm{~h}$ 且 不小于疏散时间; 机械通风系统 $120 \mathrm{~s}$ 内达 到完全运行状态; 风机在 $250^{\circ} \mathrm{C}$ 时可连续工 作 1h。美国 NFPA 130 规定小于 $61 \mathrm{~m}$ 的铁路 隧道可不设置机械通风系统, 大于 $305 \mathrm{~m}$ 的 隧道应设置; 介于 $60 \mathrm{~m}-305 \mathrm{~m}$ 之间的隧道在 完成工程分析并满足要求时可设非机械通 风系统, 如果不做工程分析或不具备非机 械通风系统设置条件的, 应设置机械通风 系统, 系统在 180s 内达到完全运行状体, 系统工作时间不小于 $1 \mathrm{~h}$ 且不小于疏散时间; 风机在 $150^{\circ} \mathrm{C}$ 时可连续工作 $1 \mathrm{~h}$ 。我国未对 通风排烟系统做出具体规定。

\section{2 .6 应急照明}

澳大利亚规定大于 $160 \mathrm{~m}$ 隧道设置应急 照明, 小于 $160 \mathrm{~m}$ 但大于 $80 \mathrm{~m}$ 隧道通过风险 评估决定是否设置, 应急照明最低照度不 应小于 $31 \mathrm{x}$, 供电时间不小于 $2.0 \mathrm{~h}$ 。欧盟 规定大于 $500 \mathrm{~m}$ 隧道设置应急照明, 最低照 度为 $11 \mathrm{x}$, 供电时间不小于 $1.5 \mathrm{~h}$ 。美国 NFPA 130 规定照度不小于 $2.71 x$ 。德国规 定大于 $1000 \mathrm{~m}$ 隧道设置应急照明, 最低照 度不小于 $1.01 \mathrm{x}$, 供电持续时间不小于 $3 \mathrm{~h}$ 。 我国规定 $5.0 \mathrm{~km}$ 及以上隧道设置应急照明, 隧道、疏散和救援通道最低照度不应小于 $0.51 \mathrm{x}$, 供电时间不应小于 $2.0 \mathrm{~h}$ 。与国外相 比, 我国对铁路隧道应急照明的规定, 无 论是设置门槛还是照度要求都低于欧美等。

\subsection{7 疏散指示标志}

我国规定 $5.0 \mathrm{~km}$ 及以上隧道设置疏散 指示标志, 间距不宜大于 $30 \mathrm{~m}$, 安装在距地 面 $1.0 \mathrm{~m}$ 以下的墙面。欧盟规定长度大于 $100 \mathrm{~m}$ 隧道设置疏散指示标志, 间距不大于 $50 \mathrm{~m}$ 。意大利规定标志设置间距不大于 $100 \mathrm{~m}$ 。 德国规定标志设置间距不应大于 $125 \mathrm{~m}$ 。法
国规定标志设置间距不大于 $100 \mathrm{~m}$ 。与国外 相比, 我国只规定 $5.0 \mathrm{~km}$ 以上的铁路隧道 设置疏散指示标志; 而欧洲国家对于 $100 \mathrm{~m}$ 以上的隧道均要求设置指示标志, 规定较 为合理; 美国无相关规定。

\section{3 地铁隧道防火关键技术指标对比}

\subsection{1 疏散与救援设施}

我国规定每段区间隧道均应设置通往 站台的疏散楼梯, 两条单线区间隧道应设 置联络通道, 联络通道间距不大于 $600 \mathrm{~m}$, 联络通道的门为甲级防火门; 美国规定双 线并行的区间隧道应设置联络通道, 通道 间距最大 $244 \mathrm{~m}$, 并规定了通道具体尺寸; 因欧洲国家双线并行区间隧道较少, 德国 等少数国家规定了双线区间隧道设置联络 通道。未见地铁区间隧道避难所相关规定。

\subsection{2 通风排烟系统}

对于隧道等密闭空间, 通风排烟系统 设置是否合理十分关键 ${ }^{[14]}$ 。我国规定地铁 区间隧道必须设置防排烟和事故通风系统; 连续长度大于 $300 \mathrm{~m}$ 的区间隧道应设置机械 防排烟设施; 连续长度位于 $60 \mathrm{~m}-300 \mathrm{~m}$ 的宜 采用自然排烟, 无自然排烟条件时设机械 排烟; 排烟量按单洞区间隧道断面排烟流 速不小于 $2 \mathrm{~m} / \mathrm{s}$ 且高于临界风速计算, 但排 烟流速不得大于 $11 \mathrm{~m} / \mathrm{s}$ 。日本规定区间隧道 应设置排烟设备, 排烟量由计算确定, 满 足自然排烟条件的可不设机械排烟。欧洲 对区间隧道排烟规定较具体, 排烟模式包 括: 隧道排烟与车站排烟相结合、仅隧道 排烟、仅车站排烟、隧道排烟与车站空调 系统相结合、车站和隧道空调系统相结合; 排烟方式包括: 坚井自然排烟、纵向排烟、 横向排烟和传感器控制排烟等。欧洲通过 差异化设计保证每条地铁的排烟方式及参 数设置均达到最优。

\subsection{3 指示标志和应急照明}

我国规定地铁区间隧道应设置疏散指 示标志, 在疏散通道拐弯处、交叉口、沿 通道长向的间距不应大于 $10 \mathrm{~m}$, 距离地面应 不大于 $1 \mathrm{~m}$; 日本规定区间隧道应设置疏散 指示标志, 间距不应大于 $100 \mathrm{~m}$, 距离地面 
$1.5 \mathrm{~m}$ 范围内。我国规定地铁区间隧道应设 置应急照明, 连续供电时间不应小于 $60 \mathrm{~min}$; 日本也规定区间隧道设置应急照明, 照度 不小于 $11 \mathrm{x}$, 连续照明时间不应小于 $1 \mathrm{~h}$; 欧洲大部分国家也规定区间隧道设置应急 照明, 均规定照度不低于 $11 x$, 其中荷兰规 定应急照度达到 $101 x^{[15]}$ 。

\section{3. 我国地铁及隧道工程标准制修订建议}

美、日、德、瑞典和澳大利亚等在地 铁及隧道防火领域发展较早, 标准体系较 为完善, 而我国无论是地铁还是公路和铁 路隧道, 其防火设计标准均无专项国家标 准。国外对隧道安全等级划分更细化, 特 别是日本和美国综合考虑多种因素, 值得 借鉴。我国隧道防火要求不够系统, 没有 明确规定公路隧道设置固定灭火设施, 而 美、日、澳和欧洲等明确提出设置要求, 并得到工程应用。对比分析提出我国在隧 道防火领域标准制修订建议:

（1）在公路隧道防火方面, 仅有行业 标准, 且多为推荐性条款, 应尽快制定公 路隧道消防设计国家标准, 明确隧道防火 等级, 特别明确隧道结构耐火、消防设施、 防排烟系统以及安全疏散等设置要求; 研 究城市公路隧道与山岭公路隧道区别, 将 各自防火性能要求纳入标准; 明确公路隧 道联络通道和紧急出口的设计参数, 增加 联络通道装饰装修等相关规定 ${ }^{[16-17]}$ 。

（2）修订《铁路工程设计防火规范》, 细化铁路隧道分级, 完善隧道防排烟技术, 提高消防设施设置要求, 系统分析设置固 定灭火系统可行性, 确定最有效的固定灭 火系统类别, 特别是明确设备洞室固定灭 火系统设置要求; 目前我国铁路隧道消防 技术标准主要适用对象为 $5 \mathrm{~km}$ 以上隧道, 与国外存在差距, 应提高消防设计要求, 可考虑将标准适用对象为 $1 \mathrm{~km}$ 以上隧道; 考虑制定铁路隧道专项防火技术标准, 并 充分吸收国外成熟标准的技术条款; 增加 铁路隧道设备洞室探测报警系统和固定灭 火系统设置要求; 系统性研究特长铁路隧 道内增设专用消防救援车的技术可行性 ${ }^{[18]}$ 。

（3）基于《建筑设计防火规范》, 研 究城市交通隧道增加固定灭火系统的可行
性及工程设置参数, 将研究成果转化应用, 形成可操作性的标准技术条款。

（4）加快《地铁设计防火规范》制定 进度, 发布前充分吸收最新防火技术成果, 保证发布时其技术水平与国外先进技术指 标保持基本一致。

（5）增加公路隧道、铁路隧道和地铁 隧道消防技术要求的适用范围, 明确不同 长度、不同类型隧道的具体消防技术要求。

\section{4. 结论}

系统性研究了国外发达国家和我国在 地铁及隧道工程防火技术领域的标准化发 展现状, 通过对比分析提出了国内外标准 化发展的技术差异, 有针对性的提出了我 地铁及隧道防火技术的发展方向和标准化 制修订内容。隧道类建筑属于复杂工程, 其安全保障技术措施体现了一个国家的综 合技术实力, 防火作为隧道类建筑的重要 技术指标, 必须投入科研力量攻克难题, 以标准化思路进行技术转化应用和并在国 际上实现在该领域的技术引领。

\section{致谢}

本研究得到了灭火救援技术公安部重点实 验室开放课题基金（KF201810）资助

\section{参考文献}

[1] 地铁设计规范, GB 50157-2013.

[2] 地铁安全疏散规范, GB/T 33668-2017.

[3] 铁路隧道设计规范, TB 10003-2016.

[4] 公路隧道设计规范, JTG D70-2-2014.

[5] 建筑设计防火规范, GB 50016-2014.

[6] 铁路工程设计防火规范,TB10063-2016.

[7] 公路隧道消防技术规范, DB43/7292012.

[8] Standard for Road Tunnels, Bridges and Other Limited Access Highways, NFPA 502-2017.

[9] Guidelines and Regulations for Road Design, RVS-2001.

[10] Minimum safety requirements for tunnels in the Trans-European road network, Directive 2004/54/EC. 
[11] Guidelines for the equipment and operation of road tunnels, RABT-2003.

[12] Tunnel fire safety, AS 4825-2011.

[13] Standard for Fixed Guideway Transit and Passenger Rail Systems, NFPA 130-2017.

[14] Liu Y, Tan C, Liu D L. CFD Analysis of Oxygen and Carbon Dioxide Recovery during Ventilation in Municipal Confined Space. Journal of Risk Analysis and Crisis Response, 2016(4):178-185
[15] Fire Protection for Tunnels, TNO 98CVB-R1161.

[16] 刘伟, 袁学勘. 欧洲公路隧道营运安全 技术的启示. 现代隧道技术, 2001, 38(1):5-10.

[17] 罗昭辉. 隧道防火灭火现状及其发展趋 势. 公路隧道, 2008(1):66-67.

[18] 刘飞. 国内铁路隧道消防技术发展探讨. 铁道勘察, 2016, 42(5):101-103. 